\section{Colloquium at the Dublin Institute for Advanced Studies}

IN July a colloquium was held at the Dublin Institute for Advanced Studies at which the following lecturers each gave a short course of lectures : Prof. C. Møller (Copenhagen), Prof. J. L. Synge (Pitts. burgh), Dr. C. F. Powell (Bristol). Prof. Møller discussed the centre of gravity in relativistic quantum mechanies, explaining how this notion can be defined in a quantized field theory and how it is related to the angular momentum and the 'trembling motion'. Prof. Synge dealt with more mathematical subjects of boundary problems and multiply connected spaces. For the non-mathematician, Dr. Powell's lectures on meson tracks in the photographic emulsion were most interesting. His and Occhialini's discovery of the existence of two kinds of mesons with different masses of which the heavier decays spontaneously into the lighter kind was widely discussed. It seemed most probable that the ordinary cosmic ray meson is identical with the lighter kind, and that at least the heavier meson is the "meson of the nuclear forces" predicted by Yukawa, and is strongly coupled with nuclei. Examples of meson emission in nuclear disintegrations which were shown in the lectures also prove that the general ideas of the meson theory are correct. It is as yet an open question whether also the light meson is strongly coupled with nuclei or not. This depends on further experiments, in particular on the question whether the mesons ejected in nuclear disintegrations are all heavy or also partly light mesons. It depends also on whether the fact that ordinary negative mesons are not captured in light elements (delayed coincidence experiments) can be understood theoretically or not. If it should turn out that the light meson is also a nuclear force meson, then the form of meson theory proposed by Møller and Rosenfeld and modified by Schwinger (vector and pseudoscalar mesons with different masses) is most likely correct.

\section{Rainfall Records of Australia}

A serious gap in the official rainfall records for Australia for the period 1939-45 would not have come as a surprise, but with the receipt recently of the 1945 issue of the official "Rain Map of Australia", it is elear that there is no such gap, but only some delay in printing. The disturbance of national life caused by the War was not allowed to affect the continuity of the 1,300 selected observing stations which are used when the official monthly and annual maps are prepared, maps which are said in the opening paragraph of the notes that accompany them to give a very fair representation of the rainfall distribution over Australia. That a number of the less. important private observing stations had to be dis. continued seems only too probable, especially in northern regions from which the population was evacuated and to which the strategy of 'scorched earth' was applied. Very brief study of the notes that accompany the rainfall maps for this last year of the War is sufficient to reveal the unstable character of the Australian climate.

In Great Britain snowstorms, floods, extremes of temperature and drought, such as those of the early part of 1947, can be fairly described as departures from 'normal' or 'average' conditions. In Australia such descriptions are almost without meaning. The 1945 notes show what happens when this fact is not taken into account. For example, the following appears under the heading "New South Wales": "In nearly every respect the year 1945 is possible of classification as a normal year. . . Rainfall was well distributed during the months of January, February, May, June, July and August, but the very heavy rains that were registered in coastal divisions during April and June caused highly destructive floods on the Northern Rivers, and enormous damage was done.... In the Richmond Basin particularly the destruction was regarded as being greater than at any time in the history of the river." So the tale goes on. Towards the end of the year, lack of rain and short bursts of heat interfered with dairying in the metropolitan zone of that State, and made a measure of rationing necessary. Grass fires were frequent. Temperatures were "not far removed from normal", yet caused eleven deaths at Broken Hill in a 7-day spell of "high readings" at inland stations. Frosts were widespread and severe during the winter and snowfall on the Alps heavier than usual. It is scarcely necessary to quote from the descriptions of weather in the regions where the weather was not described as so nearly normal ; but there is a characteristic note for Western Australia that possibly answers the natural question as to why anyone wants to live in a country with such a climate. It appears that the wheat crop there amounted to $20,500,000$ bushels, in spite of excessive winter rains and insufficient "finishing rains" in the south.

\section{Film-Strip of the Principles of Bridges}

Messrs. StripSuides, 93 Cheyneys Avenue, Canons Park, Edgware, Middlesex, have submitted a copy of StripSlide No. 47/1, "The Principles of Bridges", edited by G. Ghey, of the Royal Naval College, Dartmouth. This StripSlide consists of 35 frames. The foreword to the text which accompanies it states that "it is designed for one or two lectures whose twofold object is : (i) to give a mechanics class which has been taught the principle of moments some applications of this principle in the different types of bridges; (ii) to enable people better to appreciate a bridge when they see one and to realize how the different types are related to each other. At this level the treatment is necessarily sketchy. Though the author believes that he is telling the truth, he is well aware that it is far from being the whole truth; but if thereby the audience is encouraged to think and to ask questions the cause of education will have been furthered." The film deals picturesquely, and on the whole clearly, with shearing force, bending moment, plain beams, reinforced concrete spans, cantilever bridges and suspension bridges. Examples are shown, and the theoretical treatment, although necessarily superficial, is as adequate as can be expected in a short strip. Besides fulfilling the purpose for which it is designed, it might well be used to arouse the interest of a class in preparation for a more serious course.

\section{Archæological Research in South Africa}

RESEARCH workers are usually so busy piling brick on brick on the edifice of human knowledge that there is never time for them to stand back and survey what has been built and how it has been done. The "Loom of Prehistory" (South African Arch. Soc., Handbook Series No. 2. Pp. 152), by A. J. H. Goodwin, seeks to correct this, and attempts "some analysis of the interweaving elements of time, culture, man and area in the light of past research and publication". Students of South African pre- 